\title{
'High Resolution' Is Often Sought in SEM Imaging, But Establishing Visibility May Be the Challenge: Always Ask "What Might I Be Missing?"
}

\author{
Dale E. Newbury ${ }^{1}$ \\ ${ }^{1 .}$ National Institute of Standards and Technology, Gaithersburg, MD.
}

A constant theme spanning the history of the scanning electron microscope (SEM) has been improving spatial resolution. Recent developments in SEM instrumentation (e.g., the thermal field emission gun, low aberration objective lenses, low beam energy operation with landing energy below $1 \mathrm{keV}$, and especially contamination control) have made achieving resolution in the sub-nm range routinely possible for solving many important problems [1]. However, the challenge which is often overlooked that may limit success is the problem of establishing the "visibility" of the features of interest. Indeed, for objects that produce low contrast, the visibility problem may be so severe as to impede finding the objects of interest, especially if searching of large areas for rare objects is required. This is not a newly recognized problem. Early in the history of the SEM, a "threshold equation" for establishing the visibility of features in SEM imaging was developed, based upon the empirically-measured Rose criterion for the "average observer" to detect features in noisy images $[2,3]$. This $\mathrm{SEM}$ threshold equation relates the object contrast, $\mathrm{C}_{\mathrm{ct}}$ :

$$
\mathrm{C}_{\mathrm{ct}}=\left(\mathrm{S}-\mathrm{S}_{\mathrm{B}}\right) / \mathrm{S}
$$

where $\mathrm{S}$ is the beam-induced signal produced by a feature above the local background, $\mathrm{S}_{\mathrm{B}}$, to the critical instrument parameters, beam current $\left(\mathrm{i}_{\mathrm{B}}\right)$, pixel dwell time $(\tau)$, and overall detection efficiency $(\varepsilon$, the yield of secondary electrons (SE) or backscattered electrons (BSE) per incident beam electron multiplied by the detector collection and signal conversion efficiency):

$$
\mathrm{i}_{\mathrm{B} \text { (Threshold) }}>4 \times 10^{-18} \mathrm{~A} /\left(\mathrm{C}_{\mathrm{ct}}^{2} \varepsilon \tau\right)
$$

For given values of $\tau$ and $\varepsilon$, specifying $\mathrm{C}_{\mathrm{ct}}$ in Eqn. 2 provides an estimate of the threshold (minimum) beam current needed to establish visibility for that level of contrast. Alternatively, if the beam current is specified, Eqn. 2 gives the threshold level of contrast for which visibility can be established. Thus, for any set of instrumental parameter choices $\left(\tau, \varepsilon\right.$, and $\left.i_{B}\right)$, there will always be a threshold level of contrast below which features will not be visible. Because threshold visibility is subjective due to the acuity of the observer, Eqn. 2 should be thought of as producing imprecise "gray numbers". Threshold visibility has also been found to depend on the object size (relative to the image field) and shape [4]. For the same level of contrast, an observer is better at recognizing extended objects that cover a large fraction of the field, e.g., a fiber that crosses most of the image field, than at finding small objects, e.g., an equiaxed particle that represents only a few percent of the image area.

The threshold equation reveals that an observer's ability to discern fine spatial details will depend strongly on the level of contrast that the features produce. The smallest feature that can be discerned depends not only on the resolution limit imposed by the diameter of the focused probe and the scattering of the beam electrons within the specimen, but also on the limitations imposed by Eqn. 2. A series of images at constant beam current prepared with increasing pixel dwell time represents a traverse of contrast sensitivity and can reveal progressively lower contrast features, as shown in Fig. 1 for SEM-BSE images of the microstructure of an as-cast Ni-36Re alloy. Since there is always a threshold contrast level below which features become invisible, the absence of low contrast features in an SEM image does not guarantee that the features do not exist. It is thus challenging to prove the negative case. The prudent microscopist will always consider "What might I be missing in this image?", and perform due diligence in the form of increased pixel dwell time and/or beam current to lower the threshold contrast to a suitable value to ensure 
that features of interest really are absent. Modeling the expected contrast of a feature can provide critical information for the threshold equation that can allow informed judgment on this question.

\section{References:}

[1] J. Goldstein et al, in "Scanning Electron Microscopy and X-ray Microanalysis", (Springer, New York).

[2] C. Oatley in "The Scanning Electron Microscope", (Cambridge University Press) p. 17.

[3] A. Rose, Adv. in Electronics 1 (1948), p. 131.

[4] D. Bright, D. Newbury, and E. Steel, J. Micros. 189 (1998), p. 25.
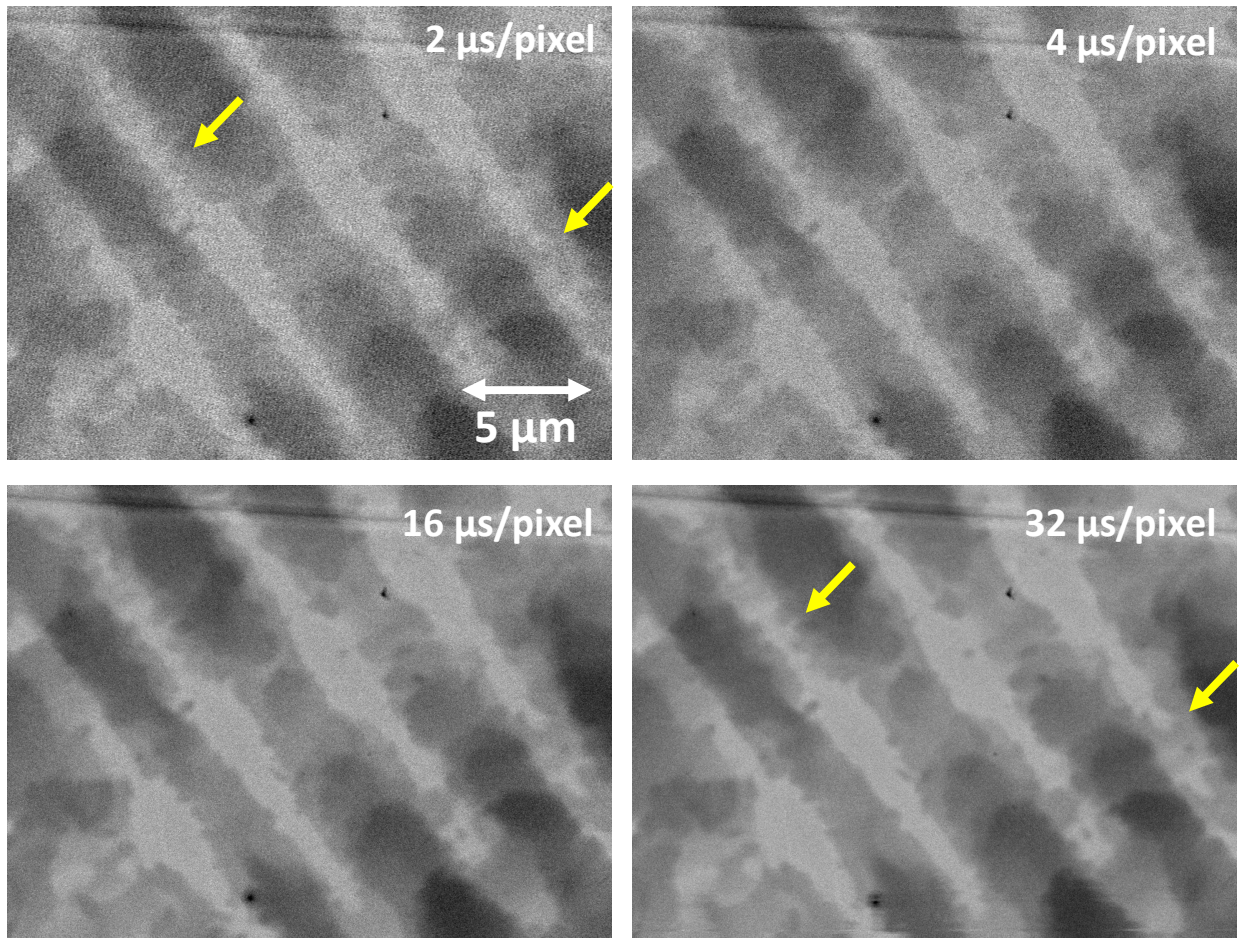

Figure 1. SEM-BSE images of Ni-36Re (as-cast) alloy showing progressive improvement in the visibility of fine scale structures as the pixel dwell time is increased (e.g., note the improved clarity of the fine scale features in the example areas near the yellow arrows). 